\title{
Radiation forces between dust grains in a plasma
}

\author{
J. T. M E D O N Ç A ${ }^{1}$ and L. STEN F L O ${ }^{2}$ \\ ${ }^{1}$ CFIF and GoLP, Instituto Superior Técnico, 1049-001 Lisboa, Portugal \\ (titomend@ist.utl.pt) \\ ${ }^{2}$ Physics Department, Umeå University, SE-90187 Umeå, Sweden
}

(Received 12 July 2007 and in revised form 28 August 2007)

\begin{abstract}
In this work we show that a repulsive force between nearby dust grains in a plasma can exist, due to scattering of the incident radiation. Two types of forces are discussed, one of them being formally identical to electrostatic repulsion. This leads to the definition of an effective dust charge of the dust grain, which only depends on the scattering process. Our discussion shows that such a repulsive interaction occurs in quite general physical conditions.
\end{abstract}

\section{Introduction}

Interaction between nearby dust particles in a plasma is an important and recurrent problem in plasma physics [1]. Various types of attractive and repulsive forces have been discussed, such as induced dipole [2], shadow forces or ion drag forces. In this paper we consider a different kind of force, due to radiation scattering. Such a force is repelling and leads to the existence of an effective electric charge of the dust grains that is different in nature from their usual electric charge.

The idea is simple and has already been considered in the context of cold atom physics [3], where it leads to observed oscillations of the confined cloud of atoms (or atomic molasses) in magneto-optical traps $[4,5]$. The same kind of phenomena can eventually occur in a dusty plasma under the action of a radiation beam. Electrostatic waves could also lead to a similar effect.

We consider a dusty plasma under the action of radiation with frequency $\omega$ and energy density $W_{0}(\omega)$. Such radiation is scattered by the dust grains. The scattering process is complex [6-8], due to separate contributions from the dust particles themselves, which leads to the well-known Mie scattering, and from the plasma electrons in the neutralizing Debye screening existing around the dust. For short wavelengths, the Mie scattering will dominate over the Debye scattering, as shown by Guerra and Mendonça [8]. However, the mechanism discussed here is independent of the detailed cross section, which only has an influence on the quantitative value of the repulsive force between nearby dust grains.

The radiation scattered by a given dust grain will interact with the nearby grains and produce a radiation pressure, which can be seen as a repulsive radiation force between grains. Such a radiation force will approximately decay with the square 
of the distance between dust grains, and can therefore be formally analogous to a repulsive force between electric charges of the same sign. Effective electric field, charge and potential can then be defined.

Here we first discuss the forces due to the incident radiation acting on a single dust grain in plasma. We then consider the secondary effects due to radiation scattering, and establish an expression for the repulsive radiation force between two nearby dust grains. Finally, we consider the case of uniform turbulence, where instead of a single incident beam of radiation we have angularly uniform incident radiation, and introduce the concept of the effective charge of a dust particle.

\section{Radiation forces on a dust grain}

We start by considering the radiation forces on a single dust grain in a plasma. The electric field associated with an incident wave with frequency $\omega$ and wavevector $\mathbf{k}$ is given by

$$
\mathbf{E}(\mathbf{r}, t)=\mathbf{E}_{0} \exp (i \mathbf{k} \cdot \mathbf{r}-i \omega t)+\text { c.c. }
$$

For simplicity, the long wavelength limit is considered, such that $k \lambda_{\mathrm{D}} \ll 1$, where $\lambda_{\mathrm{D}}$ is the Debye length. We also assume that the dust grains are spherical, with a small radius, $a \ll \lambda_{\mathrm{D}}$. These assumptions have no direct influence on the qualitative aspects of the effects to be discussed here.

Let us first consider the electron equation of motion, assuming that the ion response to the high-frequency field (1) is negligible. We can write it in the form

$$
\frac{\partial \mathbf{v}}{\partial t}=-\frac{e}{m}(\mathbf{E}-\nabla \phi)-\frac{\nabla P}{n_{0} m}-\frac{e^{2}}{2 m^{2} \omega^{2}} \nabla E^{2},
$$

where $-e$ and $m$ are the electron charge and mass, $n_{0}$ is the equilibrium plasma density, $\phi(\mathbf{r})$ is the electrostatic potential associated with the Debye screening and $P$ is the electron pressure. The first term on the right-hand side is the linear oscillating force (per unit mass) associated with the incident field and responsible for the radiation of a scattered field. The second and the third terms guarantee the maintenance of the Debye screening, and the slowly varying part of the last term describes the radiation pressure.

Let us first note that, in the absence of an incident field $\mathbf{E}_{0}=0$, the electrons will stay at rest around the dust, due to the balance between the potential and the pressure forces. Noting that the pressure depends on the local electron density inside the neutralizing region around the dust grain, $P=n(r) T$, where $T$ is the electron temperature in energy units, we obtain $\partial \mathbf{v} / \partial t=0$ for $n(r)=n_{0}[1+e \phi(r) / T]$. For, $e \phi \ll T$, this corresponds to the Boltzmann equilibrium of the electron gas in the Debye shielding potential, as given by

$$
n(r)=n_{0} \exp \left[\frac{e}{T} \phi(r)\right] .
$$

Taking the time average of the electron velocity over a period of the incident field, we get from (2) an equation of motion for a single particle belonging to the neutralizing cloud around the dust grain, as

$$
\frac{\partial}{\partial t}\langle\mathbf{v}\rangle=-\frac{\left\langle\mathbf{f}_{\mathbf{e}}\right\rangle}{m} \equiv-\frac{e^{2}}{2 m^{2} \omega^{2}} \nabla\left|E_{0}\right|^{2},
$$

where we have assumed that the Debye shielding was not significantly distorted, which is true for moderate intensities of the incident radiation. 
This slow force $\left\langle\mathbf{f}_{\mathrm{e}}\right\rangle$ pushes the neutralizing cloud in the direction of the gradient of the electromagnetic energy density. This cloud is made of an excess (or a depletion) of electrons with respect to the plasma background, for positively (or negatively) charged dust grains. In both cases, the cloud motion is due to the electron response to the ponderomotive force.

Such a slow motion is then transmitted to the dust grain itself, due to the electrostatic coupling with its neutralizing cloud. The equation of motion for the dust grain will then be

$$
M_{\mathrm{g}} \frac{d \mathbf{v}_{\mathrm{g}}}{d t}=\mathbf{F}_{\mathrm{e}}+\mathbf{F}_{\mathrm{R}}
$$

where $M_{\mathrm{g}}$ is the dust grain mass, $\mathbf{v}_{\mathrm{g}}$ its velocity, the force $\mathbf{F}_{\mathrm{e}}$ is due to the action of the incident radiation on the neutralizing cloud and $\mathbf{F}_{\mathrm{R}}$ is the radiation force acting on the dust grain itself. For the present purposes, we can use the following simple model [1]

$$
\mathbf{F}_{\mathrm{R}}=\sigma_{\mathrm{a}} W_{0} \frac{\mathbf{k}}{k}
$$

where $W_{0}=\epsilon_{0}\left|E_{0}\right|^{2} / 4$ is the energy of the incident radiation and $\sigma_{\mathrm{a}} \simeq \pi a^{2}$. On the other hand, if we assume that in its slow motion, the dust grain keeps a nearly undisturbed neutralizing could around it, we can assume that

$$
\mathbf{F}_{\mathrm{e}}=-\int\left\langle\mathbf{f}_{\mathrm{e}}\right\rangle\left[n(r)-n_{0}\right] d \mathbf{r} \simeq-\frac{Q}{e}\left\langle\mathbf{f}_{\mathrm{e}}\right\rangle
$$

where $Q$ is the dust charge, and it was assumed that the ponderomotive force $\left\langle\mathbf{f}_{\mathrm{e}}\right\rangle$ is nearly constant across the shielding region. It is also obvious that $Q e=N$ is the total electron unbalanced charge of this neutralizing region.

\section{Dust grain interactions}

Let us now consider the influence of wave scattering. The total electric field around a single dust grain in the plasma can be written as

$$
\mathbf{E}_{\text {tot }}(\mathbf{r}, t)=\mathbf{E}(\mathbf{r}, t)+\mathbf{E}_{\text {scatt }}(\mathbf{r}, t),
$$

where the incident field $\mathbf{E}(\mathbf{r}, t)$ is defined by (1) and the scattered field can be generically written as

$$
\mathbf{E}_{\text {scatt }}(\mathbf{r}, t)=\mathbf{E}_{0} \cdot \int \overline{\bar{\sigma}}\left(\mathbf{k}, \mathbf{k}^{\prime}\right) \exp \left(i \mathbf{k}^{\prime} \cdot \mathbf{r}-i \omega t\right) \frac{d \mathbf{k}^{\prime}}{(2 \pi)^{3}}+\text { c.c. }
$$

This is the total field perceived by a second dust particle, placed at a distance $R \equiv|\mathbf{r}|$ from the first. The total force on this second particle is given by expression (7) after replacing the incident field by the total field. Of course, this effect is reciprocal, and both particles will suffer a similar total force. We can see that the incident field will produce the same displacement on both particles, but the scattered field will be responsible for an additional repulsive force between nearby dust grains, as shown by the equation of motion in the frame of the first dust grain

$$
\frac{d \mathbf{v}_{\mathrm{g}}}{d t}=-\frac{2 Q e}{\epsilon_{0} m M_{\mathrm{g}} \omega^{2}} \nabla W_{\text {scatt }}+\frac{\sigma_{\mathrm{a}}}{M_{\mathrm{g}}} W_{\text {scatt }} \frac{\mathbf{R}}{R},
$$

where only the scattered radiation propagating in the direction of the vector $\mathbf{R}$, representing the position of the second grain in a reference frame centered in the 
first, is relevant to the repulsive force. In general terms, the scattered radiation intensity $W_{\text {seatt }}$ can be determined by

$$
W_{\mathrm{seatt}}=\frac{\sigma_{\mathrm{s}}}{4 \pi} W_{0} \frac{g(\theta, \psi)}{R^{2}},
$$

where $\sigma_{\mathrm{s}}$ is the total scattering cross section and $g(\theta, \psi)$ is the angular form factor for the scattered radiation. The decay in $1 / R^{2}$ is related to the scattered flux of energy across concentric spheres with radius $R$, centered at the scattering particle (the first dust grain). This shows that the repulsive force between nearby dust grains has two distinct terms. The first decays with inter-grain distance typically as $1 / R^{3}$ and is due to the ponderomotive force of the scattered radiation on the neutralizing cloud. The second decays with $1 / R^{2}$ and is due to the direct pressure of the scattered radiation on the dust grains themselves. Examples of $\sigma_{\mathrm{s}}$ can be given. For instance, in the long-wavelength limit, such that the incident wavelength $\lambda$ is much larger than both the Debye length $\lambda_{\mathrm{D}}$ and the dust grain radius $a$, we have [8]

$$
\sigma_{\mathrm{s}}=\sigma_{0}\left(\frac{Q}{e}\right)^{2}\left(\frac{1+a / \lambda_{\mathrm{D}}}{1+T_{\mathrm{e}} / T_{\mathrm{i}}}\right)^{2},
$$

where $\sigma_{0}=8 \pi r_{0}^{2} / 3$ is the Thomson cross section and $r_{0}$ is the classical electron radius. Expressions for $g(\theta, \psi)$ (as well as other expressions for $\sigma_{\mathrm{s}}$ ) can also be found in [8] or elsewhere in the literature, for instance those related to metallic clusters (see $[9,10])$. However we can see from (10) and from the above discussion that this repulsive effect between nearby dust grains or atomic clusters in a plasma is universal and is not dependent on the actual values of scattering cross sections.

For large distances, or for weakly charged dust, the force which decays with $1 / R^{2}$ is dominant. This force, associated with the direct radiation pressure of the grain, is still present if we have a collection of suspended neutral dust in a gas or in a liquid, where such repulsive effects remain. They even remain at the atomic level if instead of a collection of dust we have a collection of atoms interacting with nearly resonant incident radiation.

\section{Dust in a turbulent plasma}

Let us now extend the above results to the case of a turbulent plasma. Instead of a single incident wave with a given frequency $\omega$ we have a broad spectrum of incident radiation, where for each frequency we also have different directions of wave propagation. The case of homogeneous turbulence is particularly interesting because the direct ponderomotive and radiation forces acting on the screened dust grains cancel out, and what remains is purely spherical scattered radiation. As a consequence, the energy of the scattered radiation produced by each dust grain will be determined by

$$
W_{\mathrm{seatt}}=\frac{1}{R^{2}} \int \sigma_{\mathrm{s}}(\omega) W_{0}(\omega) \frac{d \omega}{2 \pi},
$$

where $W_{0}(\omega)$ is now the average value of the spectral energy density in the turbulent plasma, and $\sigma_{\mathrm{s}}(\omega)$ is the total scattering cross section of the dust grain. The resulting equation of motion for the dust grain placed at a distance $R$ from another 
scattering grain can then be written as

$$
\frac{d \mathbf{v}_{\mathrm{g}}}{d t}=-\alpha \nabla \frac{1}{R}-\beta \nabla \frac{1}{R^{2}},
$$

where the weighting factors for the two different forces, $\alpha$ and $\beta$, are determined by

$$
\alpha=\frac{\sigma_{\mathrm{a}}}{M_{\mathrm{g}}} \int \sigma_{\mathrm{s}}(\omega) W_{0}(\omega) \frac{d \omega}{2 \pi}
$$

and

$$
\beta=\frac{2 Q e}{\epsilon_{0} m M_{\mathrm{g}}} \int \frac{\sigma_{\mathrm{s}}(\omega)}{\omega^{2}} W_{0}(\omega) \frac{d \omega}{2 \pi} .
$$

It is interesting to compare the expression for the first force term in (14) with the repulsive electrostatic force between two particles with a fictitious charge $q_{\text {eff }}$ at a distance $R$ from each other

$$
\mathbf{F}_{\text {eff }}=\frac{q_{\text {eff }}^{2}}{4 \pi \epsilon_{0}} \frac{\mathbf{R}}{|R|^{3}} .
$$

We can then easily conclude that the first repulsive force acting on the dust particles is equivalent to a repulsive effective electrostatic force between the dust grains for an equivalent dust grain charge $q_{\mathrm{eff}}$ given by

$$
q_{\mathrm{eff}}=\left(4 \pi \epsilon_{0} \alpha\right)^{1 / 2} \text {. }
$$

This effective electric charge has no relation with the actual electric charge $Q$ of the dust particle, and it can even remain valid for a neutral dust particle if the appropriate scattering cross sections are used. It is proportional to the amplitude of the incident electromagnetic wave, and to the square root of the scattering cross section. A similar effective charge has already been identified for neutral atoms [3].

\section{Conclusions}

In this work we have shown that two nearby dust particles in a plasma repel each other in the presence of an incident electromagnetic wave. This repulsion is due to two kinds of ponderomotive forces associated with the scattered radiation. One of the forces is due to the slow response of the electrons responsible for the Debye screening and decays as $1 / R^{3}$. The other force is due to the radiation pressure on the dust grain itself and decays as $1 / R^{2}$. This second force is therefore dominant for large interacting distances and for weakly charged dust particles. It even remains for purely neutral grains in a neutral medium. This $1 / R^{2}$ force is similar to that of an electrostatic interaction between two charges, which leads us to the concept of an effective charge of the dust particles, $q_{\mathrm{eff}}$, which is proportional to the incident field amplitude and independent of the actual electric charge of the dust grains, $Q$. The case of a turbulent plasma was also discussed. This case is particularly interesting because it always leads, for homogeneous turbulence, to purely radial repulsive forces.

The concepts discussed in this work are quite general, and are qualitatively independent of the scattering processes, and of the detailed form of the scattering cross sections. Although we only have discussed the case of incident electromagnetic waves, they are also present in a variety of other situations involving electrostatic turbulence, for instance, electron-plasma or ion-acoustic turbulence. In that respect they can be related to the Casimir forces that can be defined in a turbulent 
plasma [11]. However, in contrast to the Casimir effect, the present radiation forces are always repulsive. Other related problems are the dust atom model [12], and the influence of turbulence on the Debye screening potential [13].

Finally it should be noted that the secondary radiation processes resulting from the nonlinear dynamical response of a dust particle and its neutralizing cloud to the incident radiation, such as harmonic generation $[10,14]$, would give an additional contribution to the repulsive forces discussed here.

\section{References}

[1] Shukla, P. K. and Mamun, A. A. 2002 Introduction to Dusty Plasma Physics. Bristol: Institute of Physics.

[2] Resendes, D. P., Mendonça, J. T. and Shukla, P. K. 1998 Phys. Lett. A 239, 181.

[3] Walker, T., Sesko, D. and Wieman, C. 1990 Phys. Rev. Lett. 64, 408.

[4] Labeyrie, G., Michaud, F. and Kaiser, R. 2006 Phys. Rev. Lett. 96, 023003.

[5] Pohl, T., Labeyrie, G. and Kaiser, R. 2006 Phys. Rev. A 74, 023409.

[6] Bingham, R., de Angelis, U., Tsytovich, V. N. and Havnes, O. 1991 Phys. Fluids B 3, 811.

[7] Tsytovich, V. N., de Angelis, U. and Bingham, R. 1989 J. Plasma Phys. 42, 429.

[8] Guerra, R. and Mendonça, J. T. 2000 Phys. Rev. E 62, 1190.

[9] Fomichev, S. V. and Zaretsky, D. F. 1999 J. Phys. B: At. Mol. Opt. Phys. 32, 5083.

[10] Fomichev, S. V., Popruzhenko, S. V., Zaretsky, D. F., and Becker, W. 2003 J. Phys. B: At. Mol. Opt. Phys. 36, 3817.

[11] Mendonça, J. T., Bingham, R., Shukla, P. K., and Resendes, D. P. 2001 Phys. Lett. A 289, 233.

[12] Mendonça, J. T. and Shukla, P. K. 2004 Phys. Plasmas 11, 888.

Shukla, P. K. and Mendonça, J. T. 2004 Phys. Scripta T 113, 82.

[13] Tegeback, R. and Stenflo, L. 1975 Plasma Phys. 17, 991.

[14] Tiwari, P. K. and Tripathi, V. K. 2006 Phys. Scripta 74, 682. 